\title{
LOS CONTAMINANTES ACUÍFEROS Y SUS EFECTOS NOCIVOS EN LA SALUD HUMANA
}

\author{
CAROLINA CAMpos SERRANO ${ }^{1}$
}

RESUMEN: El presente artículo tiene como objetivo evidenciar los efectos nocivos que se producen en la salud humana cuando los residuos peligrosos contaminan el agua y el medio ambiente. Estudio que se realizará mediante el análisis de la norma oficial mexicana NOM-052-SEMARNAT-2005, que establece las características, el procedimiento de identificación, clasificación y los listados de los residuos peligrosos. Por ello, y a través del método deductivo, este artículo se ha dividido en cinco secciones. Comenzando con el análisis de la citada Norma Oficial para concluir con el daño que se produce al medio ambiente, agua y la salud de los seres vivos en general y al ser humano en lo particular.

Palabras ClAve: Residuos tóxicos, contaminantes acuíferos, derecho a la salud, derecho al agua, derecho a un medio ambiente adecuado.

ABSTRACT: This article aims to evince the harmful effects that occur in human health when hazardous waste pollutes water and the environment. This study will be carried out through the analysis of the official Mexican standard NOM-052-SEMARNAT-2005, which establishes the characteristics, identification procedure, classification and lists of hazardous waste. Therefore, and through the deductive method, this article has been divided into five sections. Starting with the analysis of the Official Mexican Standard to conclude with the damage that occurs to the environment, water and the health of living beings in general and to the human being in particular.

Keywords: toxic waste, aquifer pollutants, right to health, right to water, right to the environment

1 Doctora en Derecho, profesora adscrita al Posgrado de Derecho y al Padrón de Tutores de la UNAM en los programas de Maestría y Doctorado en Derecho. 
SUMARIO: I. Introducción; II. Qué es un residuos peligroso; III. Características que definen a un residuo como peligroso; IV. Clasificación de residuos peligrosos; V. Efectos nocivos a la salud humana. VI. Conclusiones.

\section{INTRODUCCIÓN}

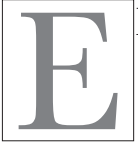

1 artículo $4^{\circ}$ de la Constitución Política de los Estados Unidos Mexicanos, entre otros, dispone la protección de los derechos fundamentales al agua, salud, y a un medio ambiente adecuado para su desarrollo integral.

Esos derechos fundamentales son necesarios para que toda persona pueda vivir en un medio ambiente adecuado para su desarrollo, salud y bienestar.

Por ello y para definir los principios de la política ambiental y los instrumentos para su aplicación; la preservación, la restauración y el mejoramiento del ambiente, se ha creado la Ley General del Equilibrio Ecológico y la Protección al Ambiente.'

De igual forma para precisar la prevención y el control de la contaminación del aire, agua y suelo, y salvaguardar el derecho de toda persona a vivir en un medio ambiente adecuado para su desarrollo y bienestar se ha creado la Ley General para la Prevención y Gestión Integral de los Residuos. ${ }^{2}$

Estos dos ordenamientos sientan las bases para la elaboración de las normas oficiales mexicanas donde se diseñan los mecanismos para el control de la contaminación del aire, agua y suelo y la calidad en términos generales para mejoramiento del ambiente y hacer posible lo dispuesto en nuestro marco constitucional.

De este modo se señala que las normas oficiales mexicanas contendrán, entre otros temas, los listados que clasifiquen los materiales y residuos peligrosos considerando sus características.

\footnotetext{
1 Ley General del Equilibrio Ecológico y la Protección al Ambiente, artículo 1.

2 Ley General para la Prevención y Gestión Integral de los Residuos, artículo 1.
} 
Es por ello que este estudio se confina al análisis de la norma oficial mexicana NOM-052-SEMARNAT-2005, que establece las características, el procedimiento de identificación, clasificación y los listados de los residuos peligrosos, la cual fue publicada el 23 de junio de 2006 en el Diario Oficial de la Federación.

\section{Qué es un RESIDUo PELIGRoso}

Los residuos se definen formalmente como los materiales o productos que se desechan ya sea en estado sólido, semisólido, líquido o gaseoso, que se contienen en recipientes o depósitos, y que necesitan estar sujetos a tratamiento o disposición final con base en lo dispuesto en la Ley General para la Prevención y Gestión Integral de los Residuos. ${ }^{3}$

Es importante señalar que un material peligroso no es lo mismo que un residuo peligroso. Al respecto al Suprema Corte de la Justicia de la Nación se ha pronunciado con la siguiente tesis:

Materiales Y Residuos Peligrosos. Su Distinción.

En términos de los artículos 3o., fracción XXIII, de la Ley General del Equilibrio Ecológico y la Protección al Ambiente y 5, fracciones XVIII, XXIX y XXXII, de la Ley General para la Prevención y Gestión Integral de los Residuos, el término "material" es definido como una sustancia, compuesto o mezcla de compuestos, cuya nota distintiva radica en que es usado como un insumo, que constituye un componente de productos de consumo, de envases, empaques, embalajes y de los residuos que éstos generan; mientras que un "residuo" es un desecho de material o producto que se encuentra en estado sólido o semisólido, o bien, en estado líquido o gas, contenido en recipientes o depósitos, y que puede ser susceptible de ser valorizado o requiere sujetar-

3 SEMARNAT. (2015) Informe sobre la situación del medio ambiente en México 2015. Compendio de estadísticas ambientales. Indicadores clave de desempeño ambiental y de crecimiento verde. Capítulo 7. Residuos, p. 431 
se a tratamiento o disposición final. ${ }^{4}$ Esto es, lo que distingue los elementos enunciados en la definición legal de materiales, de aquellos previstos en la de residuos, es que aquéllos tendrán el carácter de insumo, lo que implica la posibilidad de ser usados en un proceso productivo, en tanto que éstos se caracterizan por ser desechos, por lo que ya no pueden ser usados en algún proceso de producción. No obstante la nota distintiva anterior, ambos, en el caso que presenten características corrosivas, reactivas, explosivas, tóxicas, inflamables o biológico infecciosas, pueden considerarse como "peligrosos". En este sentido, si bien es lógico que en un proceso de producción existan residuos de los materiales -insumos-, ello no implica que pierdan este carácter, pues subsiste la posibilidad de que se utilicen en un diverso proceso y, por tanto, deben diferenciarse de los residuos -desechos-, los cuales ya fueron usados, o bien, ya no pueden usarse en el proceso de producción. ${ }^{5}$

De tal suerte que en este artículo solo se abordará lo referente a residuos peligrosos, por considerarse fuera del proceso de producción y que ya no pueden usarse nuevamente.

Los residuos peligrosos se clasifican de acuerdo a sus características y orígenes en tres grupos: residuos sólidos urbanos, residuos de manejo especial y residuos peligrosos.

En México, los Residuos Peligrosos, se definen "como aquellos que poseen al menos una de las llamadas características CRETIB, es decir, pueden ser: corrosivos $(\mathbf{C}), \operatorname{reactivos}(\mathrm{R}), \operatorname{explosivos}(\mathrm{E})$, tóxicos $(\mathrm{T})$, inflamables $(\mathrm{I})$ y biológico-infecciosos $(\mathrm{B})^{\prime \prime}$. $^{6}$

4 Énfasis agregado.

5 Gaceta del Semanario Judicial de la Federación. Tesis aislada. Décima Época. Décimo tribunal colegiado en materia administrativa del primer circuito. Libro 35, Octubre de 2016, Tomo IV. Tesis I.10o.A.29 A (10a.), Registro no. 2012896 , página: 2982

6 SEMARNAT. (2015) Informe sobre la situación del medio ambiente en México 2015. Compendio de estadísticas ambientales. Indicadores clave de desempeño ambiental y de crecimiento verde. Capítulo 7. Residuos, p.448 
Además, se consideran peligrosos "los envases, recipientes, embalajes y suelos que hayan contenido o sido contaminados con RP"7

En la parte introductoria de la Norma Oficial Mexicana NOM-052-SEMARNAT-2005 se indica que:

Los residuos peligrosos, en cualquier estado físico, por sus características corrosivas, reactivas, explosivas, inflamables, tóxicas, y biológico - infecciosas, y por su forma de manejo pueden representar un riesgo para el equilibrio ecológico, el ambiente y la salud de la población en general, por lo que es necesario determinar los criterios, procedimientos, características y listados que los identifiquen. ${ }^{8}$

Los avances científicos y tecnológicos y la experiencia internacional sobre la caracterización de los residuos peligrosos han permitido definir como constituyentes tóxicos ambientales, agudos y crónicos a aquellas sustancias químicas que son capaces de producir efectos adversos a la salud o al ambiente.

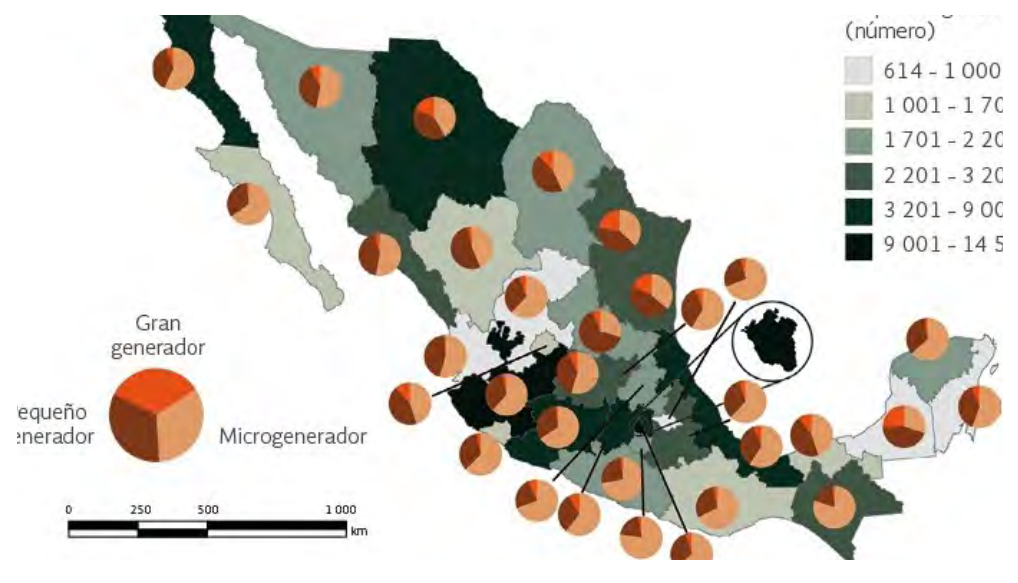

Figura 1. Empresas registradas en el Programa General de Residuos Peligrosos según tipo 2004-2014. Fuente: SEMARNAT. (2015) Informe sobre la situación del medio ambiente en México.

7 Ley General para la Prevención y Gestión Integral de los Residuos (DOF, 2003).

8 NOM-052-SEMARNAT-2005 
En la actualidad existe una gran diversidad de sustancias químicas con propósitos tan disímiles como el de utilizarse para la fabricación de nuevos materiales, medicamentos, plaguicidas, o bien como intermediarios de múltiples procesos industriales.

Sumado a lo anterior, se considera que el acelerado crecimiento de la actividad industrial en muchos países, el mal manejo de muchas sustancias químicas y la disposición inadecuada de residuos tóxicos, ha incrementado la exposición de la población y los ecosistemas naturales a sustancias potencialmente tóxicas que dañan irremediablemente al medio ambiente.

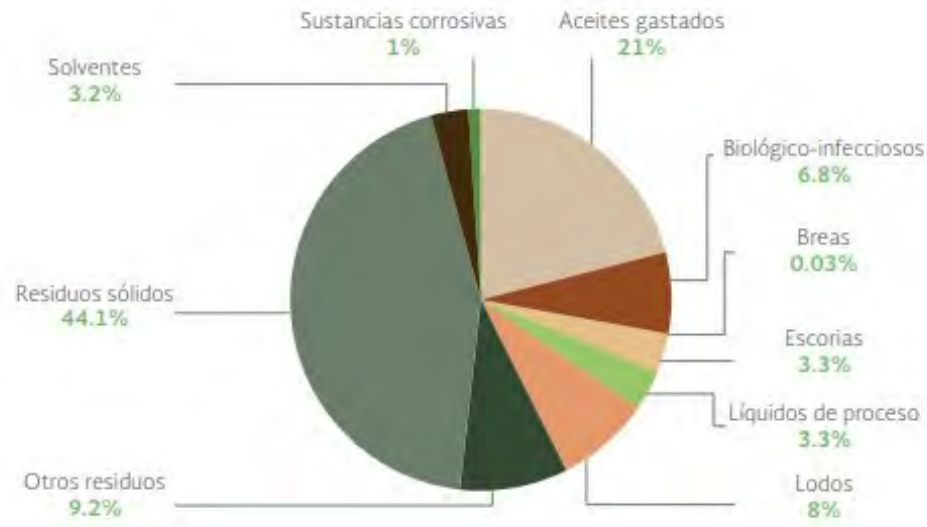

Figura 2. Generación de residuos reportada a la PGRP por tipo, 20042014. Fuente: SEMARNAT. (2015) Informe sobre la situación del medio ambiente en México. La fuente original no desagrega los materiales que integran el grupo Otros residuos.

Por todo lo anterior es que el objetivo de esta Norma es establecer el procedimiento para identificar si un residuo es peligroso.

Dentro de ese procedimiento se incluyen los listados de los residuos peligrosos y las características que hacen que se consideren como tales.

Esta norma es de observancia obligatoria en lo conducente para los responsables de identificar la peligrosidad de un residuo. 
ili. Características que definen a un residuo como PELIGROSO

El residuo es peligroso si presenta al menos una de las siguientes características:

1. Corrosividad.

2. Reactividad.

3. Explosividad.

4. Toxicidad Ambiental.

5. Inflamabilidad.

6. Biológico-Infecciosa.

En la misma Norma se indica que las Toxicidades Aguda y Crónica quedan exceptuadas de los análisis a realizar para la determinación de la característica de Toxicidad Ambiental en los residuos.

A continuación se explicará cada una de las características antes señaladas:

\section{Corrosividad}

El residuo es Corrosivo cuando una muestra representativa presenta cualquiera de las siguientes propiedades:

1. Es un líquido acuoso y presenta un $\mathrm{pH}$ menor o igual a 2,0 o mayor o igual a 12,5 .

2. Es un SóLIDO que cuando se mezcla con agua destilada presenta un pH menor o igual a 2,0 o mayor o igual a 12,5 .

3. Es un LÍQUIDO No ACUOSO capaz de corroer el acero al carbón, tipo SAE 1020, a una velocidad de 6,35 milímetros o más por año a una temperatura de $328 \mathrm{~K}\left(55^{\circ} \mathrm{C}\right)$. 
El residuo es Reactivo cuando una muestra representativa presenta cualquiera de las siguientes propiedades:

1. Es un líquido o sólido que después de ponerse en contacto con el aire se inflama en un tiempo menor a cinco minutos sin que exista una fuente externa de ignición.

2. Cuando se pone en contacto con agua reacciona espontáneamente y genera gases inflamables en una cantidad mayor de 1 litro por kilogramo del residuo por hora.

3. Es un residuo que en contacto con el aire y sin una fuente de energía suplementaria genera calor.

4. Posee en su constitución cianuros o sulfuros liberables, que cuando se expone a condiciones ácidas genera gases en cantidades mayores a $250 \mathrm{mg}$ de ácido cianhídrico por kg de residuo o $500 \mathrm{mg}$ de ácido sulfhídrico por kg de residuo.

\section{Explosividad}

El residuo es Explosivo cuando es capaz de producir una reacción o descomposición detonante o explosiva solo o en presencia de una fuente de energía o si es calentado bajo confinamiento.

Esta característica no debe determinarse mediante análisis de laboratorio, por lo que la identificación de esta característica debe estar basada en el conocimiento del origen o composición del residuo.

\section{Toxicidad Ambiental}

El residuo es Tóxico Ambiental cuando el extracto PECT, obtenido mediante el procedimiento establecido en la NOM-053-SEMARNAT-1993, contiene cualquiera de los constituyentes tóxicos listados en la Tabla 2 de esta Norma que más adelante se señalará en 
una concentración mayor a los límites ahí señalados, la cual deberá obtenerse según los procedimientos que se establecen en las Normas Mexicanas correspondientes.

\section{InFLAMABILIDAD}

El residuo es Inflamable cuando una muestra representativa presenta cualquiera de las siguientes propiedades:

1. Es un líquido o una mezcla de líquidos que contienen sólidos en solución o suspensión que tiene un punto de inflamación inferior a $60,5^{\circ} \mathrm{C}$, medido en copa cerrada, de conformidad con el procedimiento que se establece en la Norma Mexicana correspondiente, quedando excluidas las soluciones acuosas que contengan un porcentaje de alcohol, en volumen, menor a $24 \%$.

2. Es un líquido o una mezcla de líquidos que contienen sólidos en solución o suspensión que tiene un punto de inflamación inferior a $60,5^{\circ} \mathrm{C}$, medido en copa cerrada, de conformidad con el procedimiento que se establece en la Norma Mexicana correspondiente, quedando excluidas las soluciones acuosas que contengan un porcentaje de alcohol, en volumen, menor a $24 \%$.

3. No es líquido y es capaz de provocar fuego por fricción, absorción de humedad o cambios químicos espontáneos a $25^{\circ} \mathrm{C}$.

4. Es un gas que, a $20^{\circ} \mathrm{C}$ y una presión de $101,3 \mathrm{kPa}$, arde cuando se encuentra en una mezcla del 13\% o menos por volumen de aire, o tiene un rango de inflamabilidad con aire de cuando menos $12 \%$ sin importar el límite inferior de inflamabilidad.

5. Es un gas oxidante que puede causar o contribuir más que el aire, a la combustión de otro material. 


\section{BIOLÓgICO-INFECCIOSA}

El residuo es Biológico-Infeccioso de conformidad con lo que se establece en la norma oficial mexicana NOM-087-ECOL-SSA1-2002, protección ambiental-salud ambiental-residuos peligrosos biológico-infecciosos- clasificación y especificaciones de manejo.

En ella se considera un residuo biológico infeccioso cualquier microorganismo capaz de producir enfermedades cuando está presente en concentraciones suficientes (inóculo), en un ambiente propicio (supervivencia), en un hospedero susceptible y en presencia de una vía de entrada.

La norma oficial mexicana NOM-087-SEMARNAT-SSA1-2002, que establece la protección ambiental-salud, ambiental residuos peligrosos, biológico-infecciosos-clasificación y especificaciones de manejo, fue publicada en el Diario Oficial de la Federación el 17 de febrero de 2003, la cual cambió de nomenclatura por el Acuerdo Secretarial publicado en el D.O.F. el 23 de abril de 2003.

iv. Clasificación de residuos peligrosos

Los residuos peligrosos se clasifican de acuerdo a los listados que a continuación se indican:

Listado 1: Clasificación de residuos peligrosos por fuente específica.

Listado 2: Clasificación de residuos peligrosos por fuente no específica.

Listado 3: Clasificación de residuos peligrosos resultado del desecho de productos químicos fuera de especificaciones o caducos (Tóxicos Agudos).

Listado 4: Clasificación de residuos peligrosos resultado del desecho de productos químicos fuera de especificaciones o caducos (Tóxicos Crónicos). 
Listado 5: Clasificación por tipo de residuos, sujetos a Condiciones Particulares de Manejo.

Por razón del tipo de información donde se manejan fórmulas y términos químicos no se transcribirán las clasificaciones a la letra, pero sí se harán las menciones de las sustancias tóxicas y de la información más relevante que identifique al residuo contaminante peligroso.

1. Contaminantes Inorgánicos (Metales)

1. Arsénico

2. Bario

3. Cadmio

4. Cromo

5. Mercurio

6. Plata

2. Contaminantes Orgánicos semi-volátiles

1. Acido 2,4-Diclorofenoxiacético (2,4-D)

2. Acido 2,4,5 - Triclorofenoxipropiónico (Silvex)

3. Clordano

4. o-Cresol

5. m-Cresol

6. p-Cresol

7. Cresol

8. 2,4-Dinitrotolueno

9. Heptacloro (y su Epóxido)

10. Hexacloroetano 
11. Lindano

12. Metoxicloro

13. Nitrobenceno

14. Pentaclorofenol

15. Toxafeno

16. 2,4,5-Triclorofenol 400.0

17. 2,4,6-Triclorofenol 2.0

3. Contaminantes Orgánicos Volátiles

1. Benceno

2. Clorobenceno

3. Cloroformo

4. Cloruro de Vinilo

5. 1,4-Diclorobenceno

6. 1,2-Dicloroetano

7. 1,1-Dicloroetileno

8. Hexaclorobenceno

9. Hexaclorobutadieno

10. Metil etil cetona

11. Piridina

12. Tetracloroetileno

13. Tetracloruro de Carbono

14. Tricloroetileno

Listado 1. Glasificación de residuos peligrosos por fuente específica:

Giro 1: Beneficio de metales

Giro 2: $\quad$ Producción de Coque 
Giro 3: Explosivos

Giro 4: Petróleo, Gas y Petroquímica

Giro 5: Pinturas y productos relacionados

Giro 6: Plaguicidas y herbicidas

Giro 7: Preservación de la madera

Giro 8: Química farmacéutica

Giro 9 Química inorgánica

Giro 10: Química orgánica

Listado 2. Clasificación de residuos peligrosos por fuente no especifica

1. Residuos del manejo de la fibra de asbesto puro, incluyendo polvo, fibras y productos fácilmente desmenuzables con la presión de la mano. Todos los residuos que contengan asbesto el cual no esté sumergido o fijo en un aglutinante natural o artificial.

2. Todas las bolsas que hayan tenido contacto con la fibra de asbesto, así como los materiales filtrantes provenientes de los equipos de control como son: los filtros, mangas, respiradores personales y otros, que no hayan recibido un tratamiento para atrapar la fibra en un aglutinante natural o artificial.

3. Todos los residuos provenientes de los procesos de manufactura cuya materia prima sea el asbesto y la fibra se encuentre en forma libre, polvo o fácilmente desmenuzable con la presión de la mano.

4. Lodos de tratamiento de aguas residuales de apagado de las operaciones de tratamiento térmico de metales donde los cianuros son usados en los procesos. 
5. Lodos de tratamiento de aguas residuales de operaciones de galvanoplastia. En acero al carbón, Excepto de los siguientes procesos: (1) anodización de aluminio en acido sulfúrico; (2) estañado en acero al carbón; (3) zincado en acero al carbón; (4) depositación de aluminio o zinc-aluminio en acero al carbón; (5) limpieza asociada con estañado, zincado o aluminado en acero al carbón; y (6) grabado químico y acabado de aluminio depositado

6. Lodos de los baños de anodización del aluminio y lodos de tratamiento de aguas residuales del revestimiento de aluminio por conversión química

7. Residuos de los baños en operaciones de galvanoplastia donde los cianuros son usados en los procesos

8. Soluciones gastadas de baños de cianuro de las operaciones de galvanoplastia

9. Soluciones gastadas de los baños de limpieza y en operaciones de galvanoplastia donde los cianuros son usados en los procesos

10. Residuos de los baños de aceite en las operaciones de tratamiento térmico de metales

11. Soluciones gastadas de cianuros de la limpieza de tanques de baños de sal en las operaciones de tratamiento térmico de metales

12. Residuos generados en la producción de tri-, tetra- o pentaclorofenol

13. Residuos de tetra-, penta o hexaclorobenceno provenientes de su uso como reactante, producto intermedio o componente de una formulación, bajo condiciones alcalinas

14. Residuos de la producción de materiales en equipos previamente usados en la manufactura (como re- 
activo, producto químico intermedio o componente en un proceso de formulación) de tri- y tetraclorofenoles. Este residuo no incluye desechos de equipos utilizados en la producción o uso de hexaclorofeno a partir del 2,4,5triclorofenol altamente purificado. Excepto aguas residuales y carbón gastado de la purificación de cloruro de hidrogeno

15. Fondos ligeros condensados, filtros gastados y filtros ayuda y Residuos de desecante gastado de la producción de ciertos hidrocarburos alifáticos clorados a través de los procesos catalíticos de radicales libres. Estos hidrocarburos alifáticos clorados son aquellos con cadenas de uno hasta cinco carbonos y que contienen cloro en cantidades y sustituciones variadas.

16. Residuos de la producción de materiales en equipos previamente usados en la producción o manufactura de tetra-, penta- o hexaclorobencenos (como reactivo, producto químico intermedio o componente en un proceso de formulación) bajo condiciones alcalinas, excepto aguas residuales y carbón gastado de la purificación de cloruro de hidrogeno

17. Residuales de proceso, formulaciones gastadas de procesos de preservación de la madera en plantas que utilizan actualmente o hayan utilizado formulaciones de clorofenol, excepto aquellos que no hayan estado en contacto con contaminantes de proceso

18. Residuales de proceso y formulaciones gastadas de procesos de preservación de la madera en plantas que utilicen formulaciones de creosota, excepto aquellos que no hayan estado en contacto con contaminantes de proceso 
19. Residuales de proceso y formulaciones gastadas de procesos de preservación de la madera en plantas que utilicen formulaciones inorgánicas que contengan arsénico o cromo para preservar la madera, excepto aquellos que no hayan estado en contacto con contaminantes de proceso

20. Lixiviados (líquidos que han percolado a través de residuos dispuestos en tierra) resultantes de la disposición de uno o más de los residuos peligrosos antes señalados

21. Residuos resultantes de la incineración o de tratamiento térmico de suelos contaminados con los residuos peligrosos con claves:

- NE 12, producción de tri-, tetra- o pentaclorofenol

- NE 13, residuos de tetra-, penta o hexaclorobenceno

- NE 14 residuos de de tri- y tetraclorofenoles, y

- NE 16 residuos de producción de tetra-, penta- o hexaclorobencenos

Existen otras clasificaciones de residuos peligrosos.

Listado 3. Clasificación de residuos peligrosos resultado del desecho de productos químicos fuera de especificaciones o caducos

TOXICOS AGUDOS. Ver NOM-052-SEMARNAT-2005, que establece las características, el procedimiento de identificación, clasificación y los listados de los residuos peligrosos.

Listado 4. Clasificación de residuos peligrosos resultado del desecho de productos químicos fuera de especificaciones o caducos

TÓXICOS CRÓNICOS. Ver NOM-052-SEMARNAT-2005, que establece las características, el procedimiento de identificación, clasificación y los listados de los residuos peligrosos 
Listado 5. Clasificación por tipo de residuos, sujetos a condiciones particulares de manejo

1. Residuos

2. Baterias, celdas y pilas

3. Catalizadores gastados

4. Escorias

5. Lodos: acabado de metales y galvanoplastia, beneficio de metales, curtiduría, materiales plásticos y resinas sintéticas, metalmecánica, petróleo, gas y petroquímica, pinturas y productos relacionados

6. Lodos de tratamiento de aguas residuales: Acabado de metales y galvanoplastia, pilas y baterías, química inorgánica

7. Polvos: beneficio de metales, química inorgánica

8. Otros Residuos: acabado de metales y galvanoplastia, beneficio de metales, componentes electrónicos, curtiduría, explosivos, materiales plásticos y resinas sintéticas, metalmecánica, petróleo, gas y petroquímica, pilas y baterías, pinturas y productos relacionados, química farmacéutica, química inorgánica, química orgánica, textiles, varios.

9. Soluciones gastadas: Acabado de metales y galvanoplastia, beneficio de metales, componentes electrónicos, metalmecánica, preservación de la madera.

Estas son las clasificaciones respecto a los residuos peligrosos. La vigilancia de su cumplimiento le corresponde a la Secretaría de Medio Ambiente y Recursos Naturales, por conducto de la Procuraduría Federal de Protección al Ambiente. Esta última se encarga de realizar los trabajos de inspección y vigilancia que sean necesarios.

$\mathrm{Y}$, las violaciones a la misma se sancionarán en los términos de la Ley General del Equilibrio Ecológico y la Protección al Ambiente, 
la Ley General para la Prevención y Gestión Integral de los Residuos, sus Reglamentos y demás ordenamientos jurídicos aplicables.

$\mathrm{Al}$ respecto, la Suprema Corte de Justicia de la Nación reconoce como derechos fundamentales los previstos en el artículo 4 de la Constitución Política de los Estados Unidos Mexicanos:

1. Toda persona tiene derecho a la protección a la salud, [párrafo cuarto],

2. Toda persona tiene derecho a un medio ambiente sano para su desarrollo y bienestar, [párrafo quinto],

3. Toda persona tiene derecho al acceso, disposición y saneamiento de agua para consumo personal, y doméstico en forma suficiente, salubre, aceptable y asequible, [párrafo sexto]. ${ }^{9}$

En afán de garantizarlos se ha pronunciado como sigue:

MEDIO AMBIENTE. AL SER UN DEREGHO FUNDAMENTAL ESTÁ PROTEGIDO EN EL ÁMBITO INTERNAGIONAL, NAGIONAL Y ESTATAL, POR LO QUE LAS AUTORIDADES DEBEN SANCIONAR GUALQUIER INFRAGGIÓN, GONDUGTA U OMISIÓN EN SU CONTRA.

De los artículos 1 y 4 del Protocolo Adicional a la Convención Americana sobre Derechos Humanos en Materia de Derechos Económicos, Sociales y Culturales "Protocolo de San Salvador", así como el 4o., quinto párrafo, de la Constitución Política de los Estados Unidos Mexicanos, se advierte que la protección al medio ambiente es de tal importancia al interés social que implica y justifica, en cuanto resulten disponibles, restricciones para preservar y mantener ese interés en las leyes que establecen el orden público; tan es así, que en el Estado de Michoacán, la Ley Ambiental y de Protección al Patrimonio Natural del Estado, su reglamento y el Programa de monitoreo a vehículos ostensiblemente contaminantes del Estado para el año 2011, están encaminados a salvaguardar dicho derecho fundamental, proteger el ambiente, conservar el patrimonio natural, propiciar el desarrollo sustentable del Estado y establecer las bases para -entre otros

${ }^{9}$ Constitución Política de los Estados Unidos Mexicanos (DOF 2011) 
casos- tutelar en el ámbito de la jurisdicción estatal, el derecho de toda persona a disfrutar de un ambiente adecuado para su desarrollo, salud y bienestar, así como prevenir y controlar la contaminación del aire, el agua y el suelo y conservar el patrimonio natural de la sociedad. Por tanto, el derecho particular debe ceder al interés de la sociedad a tener un medio ambiente adecuado para el desarrollo y bienestar de las personas, que como derecho fundamental las autoridades deben velar, para que cualquier infracción, conducta u omisión que atente contra dicho derecho sea sancionada. ${ }^{10}$

Las sanciones aplicables no se indican en este momento por lo extenso de los supuestos que de esas leyes se desprenden.

\section{v. EfECtos nocivos A LA SALUd HUMANA}

Del estudio de esta norma se puede advertir que son muchas las fuentes que pueden contaminar al medio ambiente.

En específico para este artículo se referirá a los aparatos electrónicos, en especial las computadoras y los celulares que se convierten en una mezcla explosiva con graves consecuencias para el subsuelo y mantos freáticos.

En cuanto a los aparatos electrónicos, estos contienen metales pesados como plomo, mercurio, cadmio y berilio; químicos peligrosos como retardantes de fuego bromados, polibromobifenilos y tetrabromobisfenol. Además, usan con frecuencia polímeros con base de cloruro de vinilo.

El problema se presenta cuando los aparatos electrónicos son desechados y por un mal manejo de esos instrumentos, son convertidos en basura, conocida como basura electrónica.

"La basura electrónica o e-waste, está identificada como todo aquel desperdicio proveniente de aparatos electrónicos o eléctricos como son

10 Gaceta del Semanario Judicial de la Federación. Tesis aislada. Décima Época. Primer tribunal colegiado en materia administrativa y de trabajo del décimo primer circuito. Libro XII, Septiembre de 2012, Tomo 3. Materia Constitucional. Registro: 2001686. Tesis XI.1o.A.T.4 A (10a.), página: 1925 
computadoras, celulares, pantallas, entre otros. Este material de no ser propiamente tratado llega a la naturaleza y la contamina." 11

Ese tipo de basura y su mal manejo contaminan suelos y subsuelos de manera alarmante. La falta de información hace que las personas tiren o abandonen estos objetos indiscriminadamente los cuales su destino final son los tiraderos de basura.

La basura electrónica al liberar sustancias tóxicas en rellenos sanitarios contamina el suelo circundante, pudiendo llegar a afectar fuentes de agua subterráneas. La contaminación de suelos, pozos subterráneos y en general el ecosistema entero es resultado de la falta de conocimiento en temas de preservación de la salud ambiental.

En México solo se trata y se reutiliza un 4\% de todo el desperdicio tóxico y el resto se va directo a los tiraderos de basura y rellenos sanitarios. $^{12}$

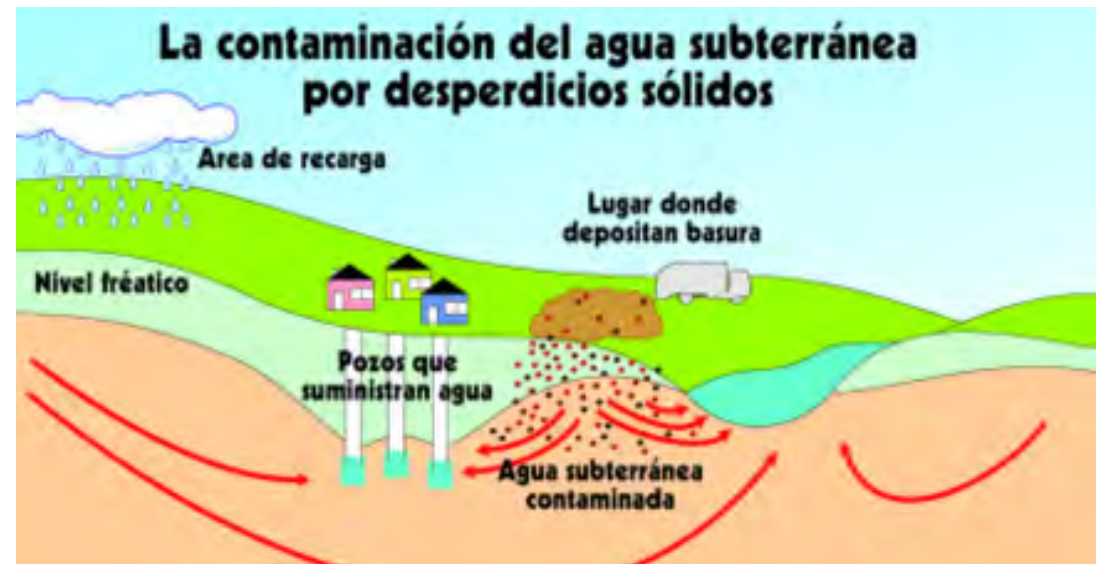

Figura 3: Contaminación del agua subterránea Fuente: Ingeniería Ambiental. Contaminación hídrica y su efecto en el medio ambiente. ${ }^{13}$

11 Fundación RET. Qué es la basura electrónica. México, 2011. Disponible en: <http://ret.org.mx/2011/01/que-es-la-basura-electronica/\#sthash.UlnNZ12H. dpuf>. Fecha de consulta: 5 julio 2014.

12 Ibidem.

${ }^{13}$ Castañeda Raúl. Ingeniería Ambiental. Contaminación hídrica y su efecto en el medio ambiente. Material didáctico para el curso de ingeniería ambiental II. Disponible: 
Por otro lado, esas sustancias al ser fundidas liberan toxinas al aire, tierra y agua convirtiéndose en algunos casos en un fuerte peligro para la salud de los seres vivos. Sin mencionar el daño gigantesco que se produce a la misma tierra incluyendo en ella a la flora y fauna del planeta.

El daño que se produce en el ser humanos es grave, por citar tres ejemplos:

1. El cromo, usado para las cubiertas de metal, es cancerígeno;

2. El cadmio, presente en la composición de baterías recargables, daña los huesos y los riñones; y

3. El mercurio, infaltable para producir iluminación en monitores, es nocivo para el sistema nervioso y el cerebro ${ }^{14}$.

\section{Efectos de la contaminación en la salud}

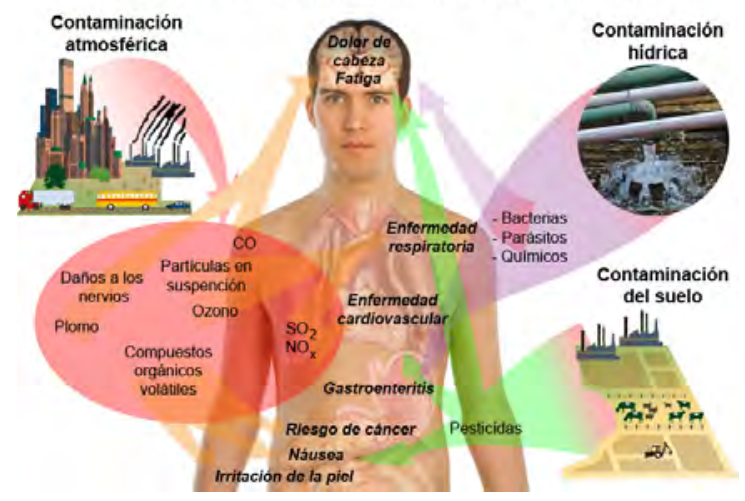

Figura 4: Efectos sobre la salud de algunos de los más típicos contaminantes. Fuente: Häggström, Mikael. Medical gallery of Mikael Häggström 2014, Wikiversity Journal of Medicine 1 (2). DOI:10.15347/ wjm/2014.008. ISSN 20018762. Public Domain.

<https://es.slideshare.net/raulcc1950/ingenieria-ambiental-contaminacin-hdri ca-y-su-efecto-en-el-medio-ambiente>. Fecha de consulta: 18 de junio 2018.

14 Michael. Desechoselectrónicos. Consecuencias que trae la basura electrónica. Disponible en: <http://desechoelectronicos.blogspot.mx/p/consecuencias-que-trae-la-basura.html>. fecha de consulta 18 de junio 2018. 
Una explicación más amplia sobre los efectos nocivos sobre la salud se encuentra en el Diagnóstico sobre la basura electrónica en México, realizado por el Instituto Nacional de Ecología y el Instituto Politécnico Nacional en el cual se muestra como la contaminación daña al medio ambiente y la salud humana. A manera de síntesis se detalla a continuación.

Tabla 1. Componentes dañinos que amenazan la salud

\begin{tabular}{|c|c|c|}
\hline Sustancia & Se encuentra en: & Daños: \\
\hline $\begin{array}{l}\text { Retardadores } \\
\text { de Flama } \\
\text { Bromados } \\
\text { (BFR) } \\
\text { Incluye a } \\
\text { los Bifenilos } \\
\text { Policlorados } \\
\text { (BPCs) y Éteres } \\
\text { bifenílicos } \\
\text { polibromados } \\
\text { (PBDEs) }\end{array}$ & $\begin{array}{l}\text { 1. Tabla de circuitería de TV } \\
\text { 2. Chasis de Plástico de TV } \\
\text { 3. TRC en TV } \\
\text { 4. Chasis de Monitor en PG } \\
\text { 5. Tarjeta de circuitos en } \\
\text { Monitor } \\
\text { 6. Plásticos y tarjetas de } \\
\text { circuitos de Teclado y ratón } \\
\text { de una PC } \\
\text { 7. Gabinete plástico en PC } \\
\text { 8. Tarjeta madre en PC } \\
\text { 9. Pasta de adhesión en el } \\
\text { microprocesador de PC } \\
\text { 10. Memoria en PC } \\
\text { 11. Plásticos de equipo de } \\
\text { videojuego } \\
\text { 12. Microprocesador de } \\
\text { videojuego } \\
\text { 13. Tarjetas de circuitos de } \\
\text { VCR } \\
\text { 14. Microcontroladores de } \\
\text { VCR } \\
\text { 15. Chasis de VCR }\end{array}$ & $\begin{array}{l}\text { Al incinerarse liberan las famosas } \\
\text { dioxinas, sustancias más tóxicas } \\
\text { conocidas por la ciencia, la } \\
\text { exposición prolongada a los } \\
\text { PBDEs es potencialmente más } \\
\text { perjudicial para la salud que la } \\
\text { exposición breve a niveles bajos de } \\
\text { PBDEs debido a la tendencia de } \\
\text { estas sustancias a acumularse en el } \\
\text { cuerpo con los años. }\end{array}$ \\
\hline
\end{tabular}


Tabla 1. Continúa

\begin{tabular}{|c|c|c|}
\hline Sustancia & Se encuentra en: & Daños: \\
\hline Plomo & $\begin{array}{l}\text { En tubos de rayo catódico y } \\
\text { soldadura }\end{array}$ & $\begin{array}{l}\text { Perturbación de la biosíntesis de } \\
\text { hemoglobina y anemia, incremento } \\
\text { de la presión sanguínea, daño a los } \\
\text { riñones, abortos y abortos sutiles, } \\
\text { perturbación del sistema nervioso, } \\
\text { daño al cerebro, disminución de la } \\
\text { fertilidad del hombre a través del } \\
\text { daño en el esperma, disminución } \\
\text { de las habilidades de aprendizaje } \\
\text { de los niños, perturbación en el } \\
\text { comportamiento de los niños, } \\
\text { como es agresión, comportamiento } \\
\text { impulsivo e hipersensibilidad, } \\
\text { alteraciones graves en la } \\
\text { propiocepción, equilibriocepción, } \\
\text { nocicepción y electrocepción, } \\
\text { magnetocepción, ecolocalización } \\
\text { en ciertos animales. }\end{array}$ \\
\hline Arsénico & $\begin{array}{l}\text { En tubos de rayo catódico } \\
\text { más antiguos }\end{array}$ & $\begin{array}{l}\text { Puedeprovocarun envenenamiento } \\
\text { rápido y la muerte. } \\
\text { El intestino, el corazón y el sistema } \\
\text { nervioso se ven afectados, cáncer } \\
\text { de piel, pulmón, vejiga o riñón. } \\
\text { La exposición en el lugar de trabajo, } \\
\text { sobre todo a través de la inhalación } \\
\text { de aire, puede causar cáncer de } \\
\text { pulmón. Fumar aumenta el riesgo } \\
\text { de este tipo de cáncer. }\end{array}$ \\
\hline
\end{tabular}


Tabla 1. Continúa

\begin{tabular}{|c|c|c|}
\hline Sustancia & Se encuentra en: & Daños: \\
\hline Selenio & $\begin{array}{l}\text { En los tableros de circuitos } \\
\text { como rectificador de } \\
\text { suministro de energía }\end{array}$ & $\begin{array}{l}\text { Puede ser fatal si no se da } \\
\text { tratamiento médico de inmediato, } \\
\text { puede causar cabello quebradizo } \\
\text { y deformidades de las uñas. En } \\
\text { casos extremos, se puede perder la } \\
\text { sensación y el control de los brazos } \\
\text { y las piernas. }\end{array}$ \\
\hline Cadmio & $\begin{array}{l}\text { En tableros de circuitos y } \\
\text { semiconductores. }\end{array}$ & $\begin{array}{l}\text { Diarreas, dolor de estómago } \\
\text { y vómitos severos, fractura de } \\
\text { huesos, fallos en la reproducción y } \\
\text { posibilidad incluso de infertilidad, } \\
\text { daño al sistema nervioso central, } \\
\text { daño al sistema inmune, desórdenes } \\
\text { psicológicos, posible daño en el } \\
\text { ADN o desarrollo de cáncer. }\end{array}$ \\
\hline Cromo & $\begin{array}{l}\text { En el acero como } \\
\text { anticorrosivo }\end{array}$ & $\begin{array}{l}\text { En niveles no letales es } \\
\text { carcinógeno, irritación de los ojos, } \\
\text { la piel y las mucosas. Provoca daños } \\
\text { permanentes en los ojos. }\end{array}$ \\
\hline Cobalto & $\begin{array}{l}\text { En el acero para estructura y } \\
\text { magnetividad. }\end{array}$ & $\begin{array}{l}\text { Vómitos y náuseas, problemas } \\
\text { de visión, problemas de corazón, } \\
\text { daño del tiroides. }\end{array}$ \\
\hline Mercurio & $\begin{array}{l}\text { Interruptores, cubiertas, } \\
\text { lámparas de bajo consumo, } \\
\text { entre otros. }\end{array}$ & $\begin{array}{l}\text { Es un veneno para el sistema } \\
\text { nervioso, afecta al sistema } \\
\text { circulatorio y al corazón. La } \\
\text { exposición durante el embarazo } \\
\text { daña el desarrollo del cerebro del } \\
\text { bebé. }\end{array}$ \\
\hline
\end{tabular}

Fuente: Dr. Guillermo J. Román Moguel (resp.), Diagnóstico sobre la basura electrónica en México, México, Instituto Politécnico Nacional/Instituto Nacional de Ecología, 2007. 
En México la población al parecer tiene poca conciencia del problema de la contaminación ambiental a través de estas sustancias tóxicas.

Una encuesta efectuada por Ipsos Mori en nueve países en 2006 indicó que en la República Mexicana apenas 43\% de mil un encuestados conocía que las computadoras personales y otros aparatos electrónicos contienen materiales dañinos para la salud humana y su entorno.

Anualmente se desechan entre 150 y 180 mil toneladas de basura electrónica, entre equipo de cómputo, televisiones, teléfonos fijos y celulares, aparatos de audio y video, sin regulación ni control. ${ }^{15}$

La tecnología se vuelve obsoleta con rapidez. Un equipo, en promedio, tiene una vida útil de tres a cinco años, por ello, es importante impulsar una cultura hacía el usuario final y la sociedad en general, para que sepa qué hacer cuando termine ese lapso.

La recomendación es acudir a dejar esta basura electrónica a centros especializados en ello para que se reciclen.

\section{vi. Conclusiones}

Los residuos peligrosos, en cualquier estado físico, presentan graves problemas al medio ambiente y a la salud del ser humano, sino son tratados con las medidas de seguridad adecuadas.

Esta realidad por sí misma es alarmante, pero la agrava el hecho de que muchos de los componentes utilizados en la fabricación de productos electrónicos son altamente tóxicos: sustancias que degradan el medio ambiente y en algunos casos son peligrosas para la salud de los seres vivos, incluido el hombre.

México se encuentra en desarrollo de una conciencia ambiental y aunque aún no se hayan logrado los resultados deseados, es im-

15 INEGI. Anuario estadístico y geográfico de los Estados Unidos Mexicanos, 2013. En base a PR. Informe de Gobierno, Anexo, Estadísticas Nacionales, Desarrollo Sustentable, (varios años), México, D.F 
portante difundir como se contamina el ambiente para evitar que se siga dañando al planeta.

Aunado a lo anterior, los efectos de la contaminación en la salud de los seres humanos es la causa de muchas enfermedades, ante lo cual se revela que no se cumple la protección a los derechos fundamentales: a la salud, a un medio ambiente sano, a una alimentación inocua, al acceso, disposición y saneamiento de agua, entre otros.

Es importante que nos detengamos a reflexionar que estamos haciendo con nuestro planeta, el medio ambiente y a la salud de todos los seres humanos.

Fuentes

Bibliografía

Castañeda Raúl, Ingeniería Ambiental. Contaminación hídrica y su efecto en el medio ambiente. Material didáctico para el curso de ingeniería ambiental II. Disponible: <https://es.slideshare.net/raulcc1950/ingenieria-am biental-contaminacin-hdrica-y-su-efecto-en-el-medio-ambiente>. Fecha de consulta: 18 de junio 2018.

Instituto Nacional de Ecología, (México, 2007) Diagnóstico sobre la basura electrónica en México. Estudio desarrollado por el Instituto Politécnico $\mathrm{Na}$ cional, Responsable: Dr. Guillermo J. Román Moguel. Disponible formato PDF.

Fundación RET, Qué es la basura electrónica, México, 2011. Disponible en: <http://ret.org.mx/2011/01/que-es-la-basura-electronica/\#st hash.UlnNZ12H.dpuf>. Fecha de consulta: 5 julio 2014.

Michael, Desechos electrónicos. Consecuencias que trae la basura electrónica, Disponible en: <http://desechoelectronicos.blogspot.mx/ p/consecuencias-que-trae-la-basura.html>, fecha de consulta 18 de junio 2018. 


\section{Estadísticas}

INEGI, Anuario estadístico y geográfico de los Estados Unidos Mexicanos, 2013. Con base en PR. Informe de Gobierno, Anexo, Estadísticas Nacionales, Desarrollo Sustentable, (varios años), México, D.F <http:// www.ine.gob.mx/descargas/diag_basura_electronica.pdf $>$.

SEMARNAT, (2015) Informe sobre la situación del medio ambiente en México 2015. Compendio de estadísticas ambientales. Indicadores clave de desempeño ambiental y de crecimiento verde. Capítulo 7. Residuos.

\section{TESIS JuRIsPrudENCIALES}

Gaceta del Semanario Judicial de la Federación. Tesis aislada. Décima Época. Décimo tribunal colegiado en materia administrativa del primer circuito. Libro 35, Octubre de 2016, Tomo IV. Tesis I.10o.A.29 A (10a.), Registro no. 2012896, página: 2982.

Gaceta del Semanario Judicial de la Federación. Tesis aislada. Décima Época. Primer tribunal colegiado en materia administrativa y de trabajo del décimo primer circuito. Libro XII, Septiembre de 2012, Tomo 3. Materia Constitucional. Registro: 2001686. Tesis XI.1o.A.T.4 A (10a.), página: 1925.

\section{LEGISLACIÓN}

Constitución Política de los Estados Unidos Mexicanos (DOF 2011)

Ley General del Equilibrio Ecológico y la Protección al Ambiente, artículo 1 .

Ley General para la Prevención y Gestión Integral de los Residuos, artículo 1 .

Ley General para la Prevención y Gestión Integral de los Residuos (DOF, 2003). 
Normas Oficiales Mexicanas

NOM-052-SEMARNAT-2005, que establece las características, el procedimiento de identificación, clasificación y los listados de los residuos peligrosos.

NOM-087-EGOL-SSA1-2002, protección ambiental-salud ambiental-residuos peligrosos biológico-infecciosos- clasificación y especificaciones de manejo. 